\title{
Architecting and Tailoring of Cell Repair Molecular Machinery: Molecule-by-Molecule and Atom-by-Atom
}

\section{Kumar R*}

National Institute of Medical Science, India

*Corresponding author: Rajiv Kumar, National Institute of Medical Science, India

Received: J anuary 18, 2020; Accepted: February 05, 2021; Published: February 12, 2021

\section{Editorial}

In cell, any breakdown in cellular (metabolic and anabolic) process because of diseases or disorders, immediately effect functioning of cell and interrupt life, art of healing (medicine) repairs cell to regain its normal functioning as earlier it was. Adverse environmental conditions responsible for damage and exceed the maintenance time of repair in cell, or insufficient repair in functioning of metabolic pathways, leads apoptosis. The endoplasmic reticulum, ER, membrane allows molecules to be selectively processed and transferred inside and outside of the cell though double-layered nuclear envelope, with the help of a pipeline between the nucleus and the cytoplasm, as well as its close association with nucleus to have a check on ribosomal translation through, a several-step process, for maintaining cellular health. Consequently, to penetrate ER equipped with such highly specific qualities, there is need to architecture and tailor of such a refined and accurate healing procedure will work selectively, effectively and precisely: molecule-by-molecule and atomby-atom [1].

For the discovery of molecular machinery capable to do repair at cellular level, an engineered architecturing and well-designed tailoring methodology must applied strategically for such task by implementing research experiences of interdisciplinary areas i.e. Drug Design and Synthesis, Cell Transport Mechanism, Nanomedicine, Molecular Modeling, Computational (Chemistry, Nanotechnology, and Spectroscopy), Atmospheric Stress, Apoptotic Pathways and Mitochondrial Involvement, DNA Damage Pathway, Toxicology, and Clinical Pharmacology). Theoretically designed machines, nanorobots, are quite feasible and will perform with the ability of repair molecule-by-molecule or atom-by-atom in cells wherein metabolic proceedings are not proper by any means. Molecules have a size, a shape, a fairly well-defined surface, and mechanical properties with the quality of natural progression which are helpful in designing, architecting and tailoring of molecular machines as smart materials [2]. The use of highly specific characterization technologies i.e. computational spectroscopy, Single-molecule localization microscopy, atomic spectroscopy: molecular spectroscopy, absorption spectroscopy and emission spectroscopy will play a key role in architecturing and tailoring of molecular machinery [3]

In nature, such molecular machinery within the natural cells persist with capacity to repair damaged cell organelles, rebuilt it, formed new cell and reassembled them. With the deeper understanding of such natural systems, feasibility of new machinery may tested with urgent efforts for designing, architecting and tailoring such frameworks particularly with the capability to repairing cells as molecule-bymolecule or atom-by-atom as demanded by cell repair and matched with necessities. Our efforts will fruitful to design new avenues after the exploitation of all resources where architecting, tailoring and manufacturing of such nano-robots will possible practically [4]. By using interdisciplinary findings, it will be much easier task to sketch the outlines of such machinery with all the capabilities operate able as per the need at molecular level with prospect of understanding of mitochondrial dynamics as living things do [5]. Then, a perfect healing methodology, working decently through molecule-by-molecule or atom-by-atom, as cell repairing mechanism will be feasible, this newly discovered approach will lead to get a breakthrough as assumed and capable to do a half-decent job by taking reactive molecules or atoms, and bringing them up to a surface in a controlled way to perform, with the addition option of making a change in it whenever and wherever required. Molecular machines will be able to sense molecular level and decide, which cell needs repair or not featuring with surgical control by remotic or magnetic regulator, molecule-by-molecule and atom-by-atom, finally, it will be a histrionic and notable breakthrough in area of medicine discovery [6-10].

Designing, architecting and tailoring of molecular machinery, with the ability to distinguish between healthy and damaged cell, is need of hour having no repercussion and with capacity of targeting effected cells or sites only selectively, effectively and precisely, developed as nano-robots, will attain great degree of understanding for cell repair or to regulate metabolic processes perfectly with controlling and diagnostics ability in-built within nano-robotic machinery which will be able to cure any disease; do repair cell; regulate normal metabolic process; control biologically originated disturbances and defects originated by any environmental factors or as a result of transcriptional mechanisms. Curing, not just alleviating, disease has always been a difficult task by any ways; the tools required for diagnosis, and knowledge of medicine which may effectively implemented to repair the affected parts or a cell. Nano-robots if designed accurately can repair at cellular level and will revolutionize the field of medicine, this may become a reality in near future.

\section{References}

1. Yeagle PL. Academic Press with the imprint of Elsevier. The Membranes of Cells. 2016.

2. Alagarsamy V. Textbook of Medicinal Chemistry. Elsevier Health Sciences 2012.

3. Mitochondria CD, Wallace RJ, Youle V. Cold Spring Harbor Laboratory Press. 2013.

4. Green DR, Reed JC. Apoptosis: Physiology and Pathology. Cambridge University Press. 2011.

5. Conn PM. Imaging and Spectroscopic Analysis of Living Cells: Imaging Live Cells in Health and Disease. Academic Press. 2012. 
6. Wang B, Hu L, Siahaan TJ. Drug Delivery: Principles and Applications. Wiley. 2016.

7. Yeo Y. Nanoparticulate Drug Delivery Systems: Strategies, Technologies, and Applications. Wiley-Blackwell. 2013.

8. Kumar N, Kumar R. Nanotechnology and Nanomaterials in the Treatment of Life-threatening Diseases. Elsevier Publication. 2013.
9. Cramer CJ. Essentials of Computational Chemistry: Theories and Models 2004.

10. Grunenberg J. Computational Spectroscopy: Methods, Experiments and Applications. 2010 